\title{
Enzymatic Quorum Quenching Using Complex Microbial System in Polymer Microcapsules Fabricated by Microfluidic Device
}

\author{
Naoyuki Takahashi ${ }^{1}$, Eri Nasuno ${ }^{1}$, Yukiko T. Matsunaga ${ }^{2}$, and Norihiro Kato ${ }^{1 *}$ \\ 1 Utsunomiya University, 7-1-2 Yoto, Utsunomiya 321-8585, JAPAN \\ 2 The University of Tokyo, 4-6-1 Komaba, Meguro-ku, Tokyo 153-8505, JAPAN \\ * Corresponding author: Fax: 81-28-689-6154, e-mail: katon@cc.utsunomiya-u.ac.jp
}

\begin{abstract}
A quorum sensing (QS) as one of the intercellular bacterial communication systems could be successfully suppressed by enzymatic degradation of QS signals, $\mathrm{N}$-acylhomoserine lactones (AHLs), inside polymer microcapsules. It is expected to isolate novel AHL-degrading bacteria and determine their AHL-degrading activity because the QS system dominates various bacterial functions including virulence expression and biofilm formation. As the QS activation index, producing red pigment prodigiosin which depends on the AHL-mediated QS in Serratia marcescens could be conveniently determined within $1-2 \mathrm{~s}$ by refractive spectrometer. Co-culture system of $S$. marcescens and model AHL-degrading Escherichia coli DH5a (pMAL-aiiA) which is genetically engineered to produce AHL-lactonase, AiiA, could successfully determine the AHL-degrading activity by measuring color change of the culture broth To prepare the microcapsules embedded with the AHL-producer and AHL-degrader, calcium alginate microgel beads could be rapidly fabricated by coaxial microfluidic device, followed by forming polyion complex with $\varepsilon$-polylysine and removing $\mathrm{Ca}^{2+}$ ions in sodium citrate solution. This microcapsule co-culture model could follow the AHL degradation by the AHL-lactonase reaction because the $a^{*}$ as the red chroma index kept negative value even after $18 \mathrm{~h}$ co-culture. This paper suggests encapsulation of the co-culture system inside hollow microcapsules which enable simultaneous evaluation of the AHL-degrading average activity for approximately 100 samples within 1-2 s.
\end{abstract}

Key words: quorum sensing, $N$-acylhomoserine lactone, microfluidic device, microcapsule

\section{INTRODUCTION}

Quorum sensing (QS) as one of the cell-cell communication systems is known as cell density-dependent mechanism to activate transcription of the QS target genes. The QS system in gram-negative bacteria is regulated by $N$-acylhomoserine lactone (AHL) which is produced as intercellular signal molecules [1]. The AHL can accumulate around cells according to cell proliferation because the AHLs are considered to diffuse through cell membranes. After reaching a threshold, the complexes of an AHL and a receptor protein stably activate expression of target genes. The AHL is popularly used to regulate various bacterial functions including bioluminescence, biofilm formation, and virulence factor production. Some opportunistic pathogens such as Pseudomonas aeruginosa and Serratia marcescens express diseases by mediating the QS. Thus, blocking bacterial infectious diseases becomes possible without antibiotics by keeping a low AHL concentration to inhibit the QS. Inactivation of the various AHLs is a novel effective strategy for defense against disease with avoiding the appearance of antibiotic-resistant bacteria. In this research, enzymatic degradation of the AHL was carried out in polymer microcapsules.

Quorum quenching due to AHL-acylase or AHL-lactonase produced by some bacterial cells has been reported [2, 3]. The AHL-lactonase opens the lactone ring of AHL, while the AHL-acylase hydrolyzes the amide bond and separates into organic acid and homoserine lactone. An AHL-lactonase gene aiiA was cloned from Bacillus cereus ATCC14579 [4], and AiiA was expressed as a fusion protein with $N$-terminal maltose binding protein (MBP) tag in Escherichia coli DH5 $\alpha$. The genetically modified E. coli (pMAL-aiiA) was employed as a model AHLdegrader to inactivate the AHLs produced by the model AHL-producer $S$. marcescens AS-1.

In $S$. marcescens AS-1, $N$-hexanoyl-Lhomoserine lactone (C6HSL) and $\mathrm{N}$-(3-oxohexanoyl)-L-homoserine lactone (3O-C6HSL) are mainly produced to express pig clusters regulating production of a red pigment, prodigiosin (Fig. 1). Activation of the QS can be visually determined by changing color of the cell suspension due to accumulation of prodigiosin [5] By co-culturing AHL-degrading bacteria and $S$. marcescens, the suppressive effect of the 


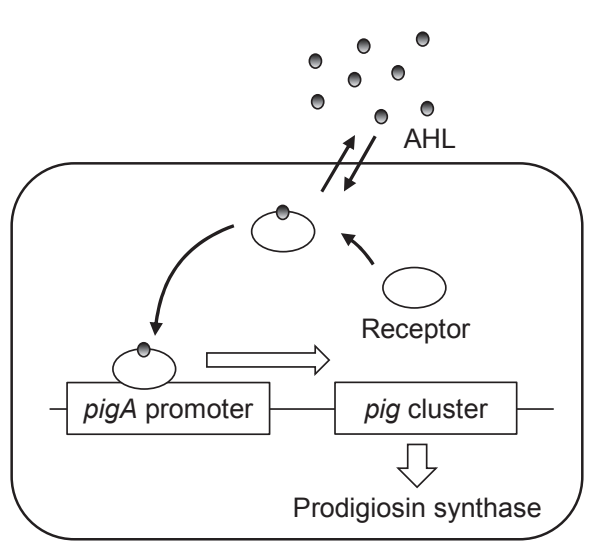

Fig. 1 Illustration of the mechanism for prodigiosin production mediated by AHLdependent quorum sensing in Serratia marcescens AS-1. After increasing the AHL concentration above a threshold, red pigment prodigiosin is produced and accumulated inside cells.

AHL-hydrolase on the AHL-mediated QS in $S$. marcescens could be determined by detecting color change. Furthermore, non-destructive method of collecting cultured cell enabled quick detection within 1-2 s by measuring reflected light spectra, while the conventional method needs approximately $30 \mathrm{~min}$ only for one sample due to extracting prodigiosin.

Both model AHL-producer and -degrader cells embedded in polymer microcapsules were co-cultured to simultaneously determine the AHL-degrading average activity for 100 samples (Fig. 2). The chroma at the microgel surface could be automatically plotted as a color coordinate on $C I E-L^{*} a^{*} b^{*}$ color space specified by Commission International de I'Eclairage (CIS) in 1976.

The AHL-producer and -degrader were encapsulated into calcium alginate microgel beads fabricated by co-axial microfluidic device. The $G$ blocks consisted of $\alpha$-L-guluronic acid in the sodium alginate were crosslinked with divalent cations such as $\mathrm{Ca}^{2+}$. The hollow microcapsules created by removing $\mathrm{Ca}^{2+}$ ions from the gel beads were immersed in Luria-Bertani (LB) liquid medium to co-culture the AHL-producer and the AHL-degrader inside the capsules.

The objective of this research is to fabricate uniformly sized polymer microcapsules embedded with the E. coli expressing the AHL-lactonase AiiA and $S$. marcescens AS-1 to construct the co-culture system. By using refractive spectrometer, simultaneous determination of AHL-degrading average activity for approximately 100 samples was demonstrated as the novel technique.

\section{EXPERIMENTAL}

\subsection{Materials}

Sodium alginates (I-1G, IL-6) were purchased from KIMICA Co. (Tokyo, Japan). Calcium

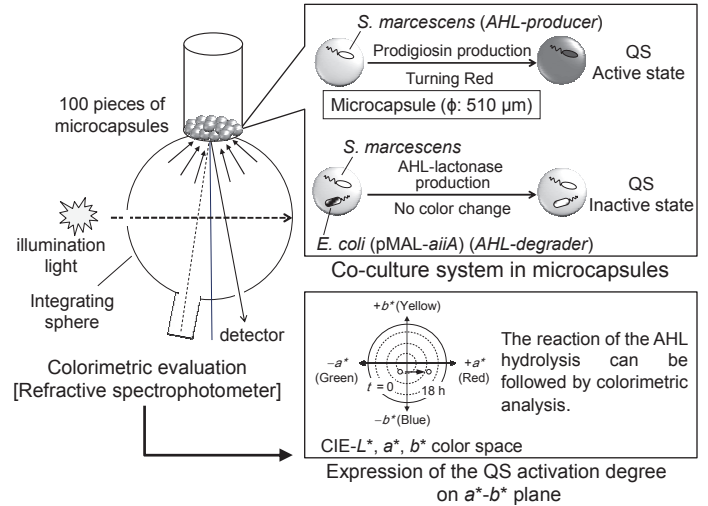

Fig. 2 Quick detection of the AHL-degrading activity in microcapsule co-culture system using refractive spectrometer. The AHLdegrading average activity of approximately 100 samples can be given within 1-2 s.

carbonate, lecithin, acetic acid, and tri-sodium citrate were obtained from Kanto Chemical Co. (Tokyo, Japan). Corn oil, $n$-hexadecane, and $\varepsilon$-polylysine were purchased from Wako Pure Industries Co., Tokyo Chemical Industry Co., and JNC Co., respectively.

\subsection{AHL-lactonase activity of AiiA}

Hydrolysis reaction of $10 \mu \mathrm{M}$ C6HSL by AiiA extracted from the recombinant $E$. coli was carried out at $30^{\circ} \mathrm{C}$ in the presence of $17.5 \mu \mathrm{M}$ MBP-AiiA in $67 \mathrm{mM}$ phosphate buffered saline (pH7.4). After the desired period, the mixture was heated to $90^{\circ} \mathrm{C}$ for $5 \mathrm{~min}$ to stop the hydrolysis by inactivating AiiA. The AHL-lactonase activity was demonstrated by using the well-known and sensitive AHL reporter strain Chromobacterium violaceum CV026 which is an AHL-synthase defective mutant and produces a purple pigment violacein via the QS system by only adding AHLs from outside cells [6]. AHL detection is easily enabled by appearing a purple spot when C6HSL solution is spotted onto filter papers $(\Phi=8 \mathrm{~mm})$ put on the agar plate containing CV026 cells. Each $20 \mu \mathrm{L}$ of the C6HSL solution with or without AiiA was spotted onto the filter paper. Difference between diameters of the purple spots was observed after culture at $30^{\circ} \mathrm{C}$ for $12 \mathrm{~h}$ to determine the amounts of undegraded C6HSL in the mixture.

\subsection{Prodigiosin production assay}

S. marcescens AS-1 was precultured in LB liquid medium at $25^{\circ} \mathrm{C}$ for $24 \mathrm{~h}$. The turbidity of the preculture broth at $600 \mathrm{~nm}\left(O D_{600}\right)$ was adjusted to approximately 6.0 by adding fresh LB medium. Recombinant E. coli $\mathrm{DH} 5 \alpha$ harboring aiiA gene showed high C6HSL-degrading activity. The culture broth turbidity of the AHL-degrading E. coli precultured at $37^{\circ} \mathrm{C}$ in LB broth containing $100 \mu \mathrm{g} / \mathrm{mL}$ ampicillin was condensed to adjust to approximately 6.0 . The preculture solution of $S$. marcescens AS-1 was inoculated in 


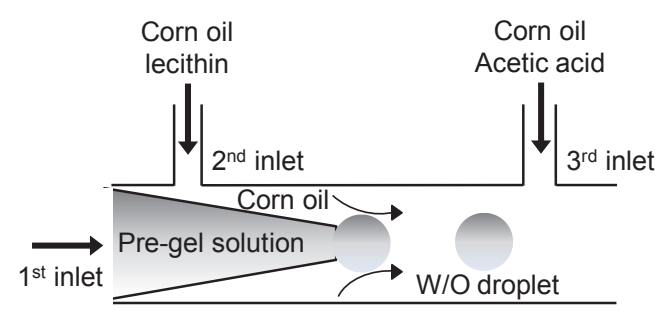

Fig. 3 Schematic view of the microfluidic device. The pre-gel solution through the $1^{\text {st }}$ inlet is aqueous sodium alginate solution suspended with $\mathrm{CaCO}_{3}$ particles, AHLproducer cells, and AHL-degrader cells. Through $2^{\text {nd }}$ and $3^{\text {rd }}$ inlets, corn oils containing lecithin and acetic acid are flown, respectively.

$4 \mathrm{~mL}$ LB liquid medium at $1 \%$ and $E$. coli (pMAL-aiiA) simultaneously inoculated at 1, 2, 5, or $10 \%$ as the desired volume ratio (S. marcescens/E. coli) from $1 / 1$ to $1 / 10$. After shaking culture at $30^{\circ} \mathrm{C}$ for $15 \mathrm{~h}$, production of the QS-dependent prodigiosin was conventionally determined as follows [7]. The culture broth was centrifuged, and then the cell pellet was re-suspended in acidified ethanol to extract the prodigiosin from cells. After the centrifugation again, the absorbance at $534 \mathrm{~nm}$ of the supernatant $\left(A_{534}\right)$ was measured as the maximum absorption wavelength of the red colored prodigiosin.

\subsection{QS suppression test in microcapsules}

To demonstrate the quorum quenching, two different bacteria as an AHL-producer (S. marcescens) and an AHL-degrader (E. coli) were embedded into polymer microcapsules prepared by co-axial microfluidic device [8]. The 2 wt $\%$ sodium alginate aqueous solution (I-1G) containing 0.75 wt $\%$ micronized calcium carbonate and both bacterial cells with an appropriate volume ratio flows into the inner channel of the device at $0.30 \mathrm{~mL} / \mathrm{h}\left(1^{\text {st }}\right.$ inlet $)$. The corn oil containing $2 \mathrm{wt} \%$ lecithin flows into the outer channel $\left(2^{\text {nd }}\right.$ inlet $)$ to form water-in-oil $(\mathrm{W} / \mathrm{O})$ droplets. The pre-gel solution was broken into droplets at the orifice by flow of the corn oil. The alginate $\mathrm{W} / \mathrm{O}$ droplets were steadily gelled by free $\mathrm{Ca}^{2+}$ ions when the $\mathrm{pH}$ was decreased by means of mixing with the other corn oil containing $1.5 \mathrm{wt} \%$ acetic acid poured from the $3^{\text {rd }}$ inlet (Fig. 3). After washing the corn oil by $n$-hexadecane, outer surfaces of calcium alginate gel beads were coated by alternate immersion in aqueous solution of $0.1 \mathrm{wt} \%$ polylysine containing $0.85 \mathrm{wt} \%$ calcium chloride and 0.1 $\mathrm{wt} \%$ sodium alginate (IL-6). The gel beads with 7 layers of polyion complexes were immersed into $0.5 \mathrm{wt} \%$ tri-sodium citrate solution for $1 \mathrm{~h}$ to dissolve alginate matrix inside the gel beads. Hollow structure of the polymer capsules was confirmed by microscopic observation of the cracked capsules. About 400 to 500 capsules

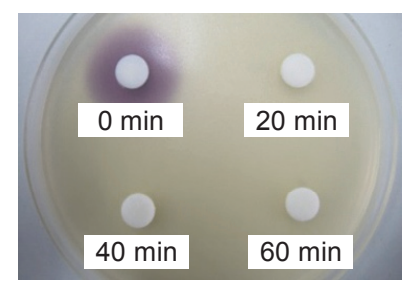

Fig. 4 Inhibition of QS-dependent violacein production due to hydrolyze of $10 \mu \mathrm{M}$ C6HSL by $17.5 \mu \mathrm{M}$ MBP-AiiA. After hydrolysis at $30^{\circ} \mathrm{C}$ for $0,20,40$, or $60 \mathrm{~min}$, $20 \mu \mathrm{L}$ of each hydrolyzed solution was spotted onto the filter paper and incubated at $30^{\circ} \mathrm{C}$ for $12 \mathrm{~h}$.

containing the AHL-producer and-degrader were added into LB liquid medium and cultured with gentle shaking at $30^{\circ} \mathrm{C}$ for $18 \mathrm{~h}$. Color change of the microcapsules is a convenient index for quenching the QS because the hydrolysis of AHLs prevents changing color of the microcapsules to red. The color change of culture broth containing the microcapsules embedded with only $S$. marcescens AS-1 (control) or both $S$. marcescens AS-1 and E. coli (pMAL-aiiA) was colorimetrically analyzed by spectrophotometer (SD-6000, Nippon Denshoku Co.). The colors based on reflected light spectra were evaluated by color coordinates in the CIE- $L * a * b *$ color space $[9,10]$. In the CIE- $L * a * b *$ color space, brightness and chroma are represented with $L^{*}$ and $a^{*} b^{*}$ coordinates, respectively.

\section{RESULTS AND DISCUSSION}

3.1 Quorum quenching by AHL-lactonase

To investigate the AHL-lactonase activity, C. violaceum CV026 assay was carried out. The hydrolysis reaction of C6HSL proceeded in the presence of AHL-degrading bacterium for 20-60 min. No purple spot appeared even after $20 \mathrm{~min}$ reaction, while $10 \mu \mathrm{M}$ C6HSL control successfully induced the QS-dependent violacein production (Fig. 4). The violacein production in CV026 cells are known to be induced by AHLs possessing $\mathrm{N}$-acyl side chains from $\mathrm{C} 4$ to $\mathrm{C} 8$ in length [11]. Thus, it is reasonable to determine whether the QS in CV026 cells is quenched by AiiA. These results clearly showed that the quorum quenching was enabled by inactivation of the C6HSL in the presence of AHL-degrading bacteria.

Accordingly, S. marcescens AS-1 and E. coli DH5 $\alpha$ (pMAL-aiiA) were co-cultured in different mixing ratios. As shown in Fig. 5, the relative prodigiosin production (RPP) decreased with increasing the ratio of AHL-degrading bacteria. By mixing a 10 -fold excess of the initial E. coli cells, production of the AHL-mediated prodigiosin became almost zero. As expected, catalyzing hydrolysis of the AHL was also effective to quench the sequential QS system when the AHL-degrading bacteria were 


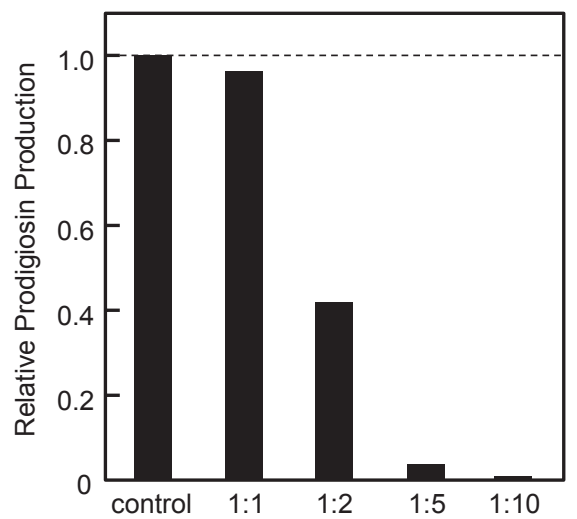

Turbidity ratio (S. marcescens AS-1 : E. coli DH5a)

Fig. 5 Suppressive effects of E. coli DH5 $\alpha$ on AHL-mediated prodigiosin production in S. marcescens AS- 1 . Co-culturing at $30^{\circ} \mathrm{C}$ for $15 \mathrm{~h}$
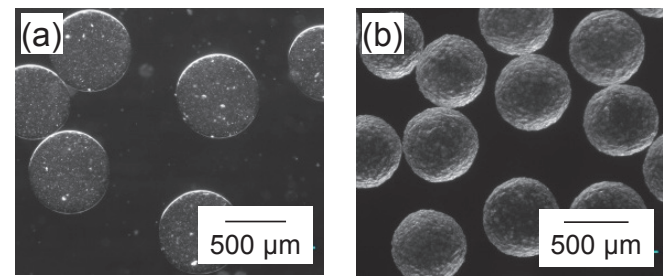

(c)

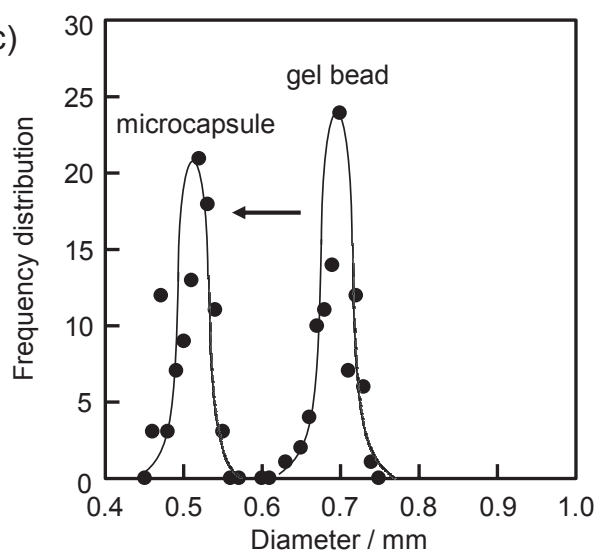

Fig. 6 The characterization of the calcium alginate micogel beads and polyion complex microcapsules. The microscope images of (a) microgel beads and (b) microcapsules. (c) A shift towards smaller diameter by forming hollow capsules.

co-cultured with $S$. marcescnes that produces C6HSL and 30-C6HSL.

3.2 Hollow capsule manufacture by co-axial microfluidic device

Calcium alginate microgel beads containing both $S$. marcescens AS-1 and E. coli DH5a (pMAL-aiiA) were produced by using a co-axial microfluidic device. Alternate polyion layers at the microgel surfaces were prepared by repeating alternate immersion in solutions between cationic polylysine and anionic alginate. To manufacture

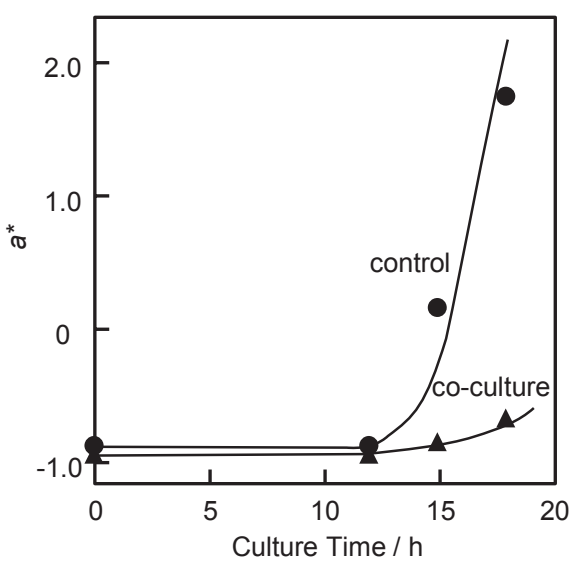

Fig. 7 Time evolution of prodigiosin production inside microcapsules evaluated in the $L^{*} a^{*} b^{*}$ color space. Average $a^{*}$ value of approximately 100 capsules could be simultaneously determined. Closed circles represent an increase of $a^{*}$ in the case when the microcapsules contained solely with $S$. marcescens AS-1 (control). Closed triangles represent $a^{*}$ during co-culturing in the case when the microcapsules contained both S. marcescens AS-1 and E. coli

the hollow micocapsules, excess alginate was eluted by citrate treatment. The microscope images of the microgel beads and hollow microcapsules were respectively shown in Fig. 6a and $b$. Size distributions of the microgel beads and microcapsules were determined by measuring diameter of 100 samples under the stereo microscope (M165C, Leica). The average diameter and the coefficient of variation (C.V.) of microgel beads were respectively $690 \mu \mathrm{m}$ and $3.9 \%$, while those of microcapsules were respectively $510 \mu \mathrm{m}$ and $4.7 \%$. These results suggested that the bead size slightly shrank during forming polyion coating, and besides the monodispersity was maintained during hollowing process (Fig. 6c)

\subsection{Quorum quenching by AHL-lactonase}

The microcapsules containing the AHLproducer and-degrader were immersed in LB liquid medium at $30^{\circ} \mathrm{C}$. The degree of the QS activation in $S$. marcescens AS-1 was monitored when both bacteria inside the microcapsules were cultured with shaking the microcapsules. Time evolution of the color change of the microcapsules was followed by reflectance measurement and then the chromas of the microcapsules in the liquid broth were evaluated in CIE- $L * a * b *$ color space, while the increase in $a^{*}$ value from the center of the color space toward the plus or minus directions shows increases in red or green chroma, respectively. Color change of the microcapsules during the process of prodigiosin production preferentially lead to change $a^{*}$ value. Figure 7 shows the 
change in $a^{*}$ with increasing time, when the co-culture system was investigated in polymer microcapsules. By embedding the AHL-degrading bacteria, the increase of the $a^{*}$ value was suppressed throughout the $18 \mathrm{~h}$ culture. This result clearly shows that the QS could be inhibited inside the microcapsules due to hydrolyzing AHL signals, and also inactivation effect of the AHL-lactonase could be quickly measured as the 100 sample average using refractive spectrometer.

\section{CONCLUSIONS}

By embedding both model AHL-producing bacteria, $S$. marcescens AS-1, possessing the gene pig clusters that code prodigiosin synthase, and model AHL-degrading bacteria, E. coli DH5a (pMAL-aiiA), possessing the gene aiiA that codes AHL-lactonase, the QS-dependent prodigiosin production in $S$. marcescens could be successfully blocked inside the microcapsules. Relatively monodispersed microcapsules were easily fabricated by co-axial microfluidic device even when two model bacterial cells were embedded for co-culture. The microcapsule co-culture system has high potential to quickly determine the AHL-degrading activity of the coexisted bacteria because refractive analysis (light transmission area: $\Phi \quad 6.4 \mathrm{~mm}$ ) enables simultaneous measurement for multiple culture broth partitioned by microcapsules $(\Phi 0.5 \mathrm{~mm})$.

\section{ACKNOWLEDGEMENT}

This work was supported by JSPS KAKENHI Grant Number 24560956.

\section{REFERENCES}

[1] E. P. Greenberg, ASM News, 63, 371-377 (1997).

[2] Yi-Hu Dong, Jin-Ling Xu, Xian-Zhen Li, and Lian-Hui Zhang, Proc. Natl. Acad. Sci. USA., 97, 3526-3531 (2000).

[3] Jared R. Leadbetter and E. P. Greenberg, $J$. Bacterial., 182, 6921-6926 (2000).

[4] Yi-Hu Dong, Andi R. Gusti, Qiong Zhang, Jin-Ling Xu, and Lian-Hui Zhang, Appl. Environ. Microbiol., 68, 1754-1759 (2002).

[5] N. Kato, A. Kobayashi, H. Motohashi, Y. Ozonoe, T. Morohoshi, and T. Ikeda, Progr. Colloid Polym. Sci., 136, 155-162 (2009).

[6] K. H. McClean, M. K. Winson, L. Fish, A. Taylor, S. R. Chhabra, M. Camara, M. Daykin, J. H. Lamb, S. Swift, B. W. Bycroft, G. S. Stewart, and P. Williams. Microbiology, 143, 3703-3711 (1997).

[7] H. Slater, M. Crow, L. Everson, and G. P. C. Salmond, Mol. Microbiol., 47, 303-320 (2003).

[8] Y. Morimoto, Wei-Heong Tan, S. Takeuchi, Biomed Microdevices, 11, 369-377 (2009).

[9] R. D. Douglas and M. Przybylska, J. Prosthet. Dent., 82, 143-149 (1999)

[10] E. Nasuno, C. Okano, K. Iimura, T. Morohoshi, T. Ikeda, and N. Kato, Mater. Res. Innov., 18, S879-S883 (2014).
[11] K. H. McClean, M. K. Winson, L. Fish, A. Taylor, S. R. Chhabra, M. Camara, M. Daykin, J. H. Lamb, S. Swift, B. W. Bycroft, G. S. A. B. Stewart, and P. Williams, Microbiology, 143, 3703-3711 (1997).

(Received May 30, 2014; Accepted August 25, 2014) 